\title{
Incidental primary peritoneal serous borderline tumor miming endometriosis during laparoscopy for chronic pelvic pain
}

\author{
Linda Tebache $^{1 *}$, Géraldine Brichant ${ }^{1}$, Claire Paquet ${ }^{1}$, Frédéric Goffin ${ }^{1}$, Katty Delbecque ${ }^{2}$ and Michelle Nisolle $^{1}$ \\ ${ }^{1}$ Department of Obstetrics and Gynaecology, University of Liège, Belgium \\ ${ }^{2}$ Pathology Department, University of Liège, Belgium
}

Keywords: endometriosis, chronic pelvic pain, primary peritoneal serous borderline tumor, laparoscopy

\section{Background}

Typically, multifactorial, chronic pelvic pain (CPP) is a common complaint in woman of childbearing age. In such case, laparoscopy as a diagnostic tool, allows also to treat certain pathologies related to $\mathrm{CPP}$, like endometriosis (presence of uterine endometrial tissue outside of the normal location) [1]. Complete surgical excision of endometriosis has been proved as an effective treatment to relieve pain and improve quality of life [2]. Even if in most cases, endometriotic lesions appeared like purple or blue nodules during laparoscopy, they may have atypical aspects in 15-30\% of patients (peritoneum opacification or circular defects, glandular growths, inflammatory alterations, yellow spots, ovarian adhesions) that could be mistaken for other non-endometriotic lesions, such as endosalpingiosis or primary peritoneal serous borderline tumor (PPSBT) $[3,4]$.

Endosalpingiosis differs histologically from endometriosis and refers to the presence of ectopic, cystic glands lined with ciliated epithelium similar to that found in the normal endosalpinx [5].

PPSBT is a rare entity also known as serous micropapillomatosis of low malignant potential and previously designated as 'atypical endosalpingiosis'. It appears to be a proliferation of epithelial cells arising from secondary müllerian system remnants within or close to the peritoneum. Although PPSBT are usually an incidental finding: multiples granules, nodules or plaques are visible on the peritoneum with the possible existence of adhesions; some lesions have been associated with abdominal or pelvic pain [6]. PPSBT can trigger suspicion for a primary ovarian tumor with peritoneal implants and therefore both ovaries must be checked carefully directly or by imaging before the diagnosis of PPSBT can be confirmed [7,8]. The prognosis for patients with PPSBT is very good, but the low risk of recurrence or of progression to a low-grade peritoneal serous carcinoma, justify a long-term follow-up. Removal of as many of the peritoneal lesions as possible is the treatment of choice, with a possibility of conservative surgery, for patient who want to conceive. Retrospective series have reported that there were no significant benefits from postoperative adjuvant therapy $[6,9,10]$.

\section{Case Report}

A 26-year-old female patient was referred to our CPP center because of failure of any medical treatment for dysmenorrhea. Clinical and imaging examinations including pelvic ultrasounds and magnetic resonance imaging were unremarkable. We decided to perform an exploratory laparoscopy to exclude undiagnosed endometriosis or any other abnormalities linked to CPP. During the surgery a few endometriosis-like implants were discovered on the vesico-uterine peritoneum (Figure 1). Samples were sent for anatomopathological analyses and the other lesions were vaporized by a $\mathrm{CO}_{2}$ laser. Day hospital was uneventful. Surprisingly, final analyses were consistent with serous borderline non-invasive peritoneal implants (primary peritoneal serous borderline tumor: PPSBT) (Figure 2).

As a result, a second look laparoscopy was planned. Preoperative thoracic and abdominal CT-scan did not identify any suspicious lesions neither adenopathy. Multiple random peritoneal, ovarian biopsies and omentectomy were performed for new histological lecture. Postoperative recovery was uneventful, and the patient was discharged home the day after. Subsequent analysis revealed no other positive PPSBT implants but identified endometriosis (Figure 3) and endosalpingiosis (Figure 4). No adjuvant treatment was recommended. At review 5 weeks later, she was feeling well and complaining of less dysmenorrhea. The patient is being followed-up with no new events at 19 months. 


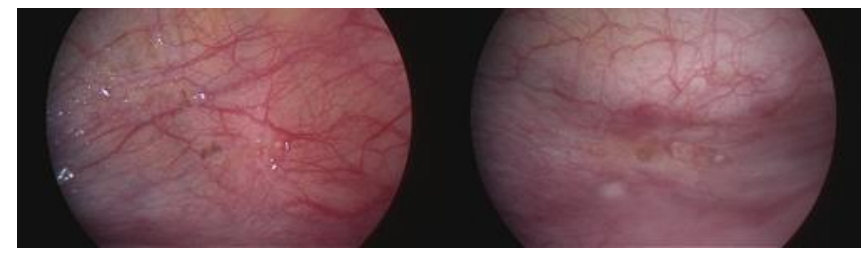

Figure 1. Laparoscopic view of endometriosis-like PPSBT

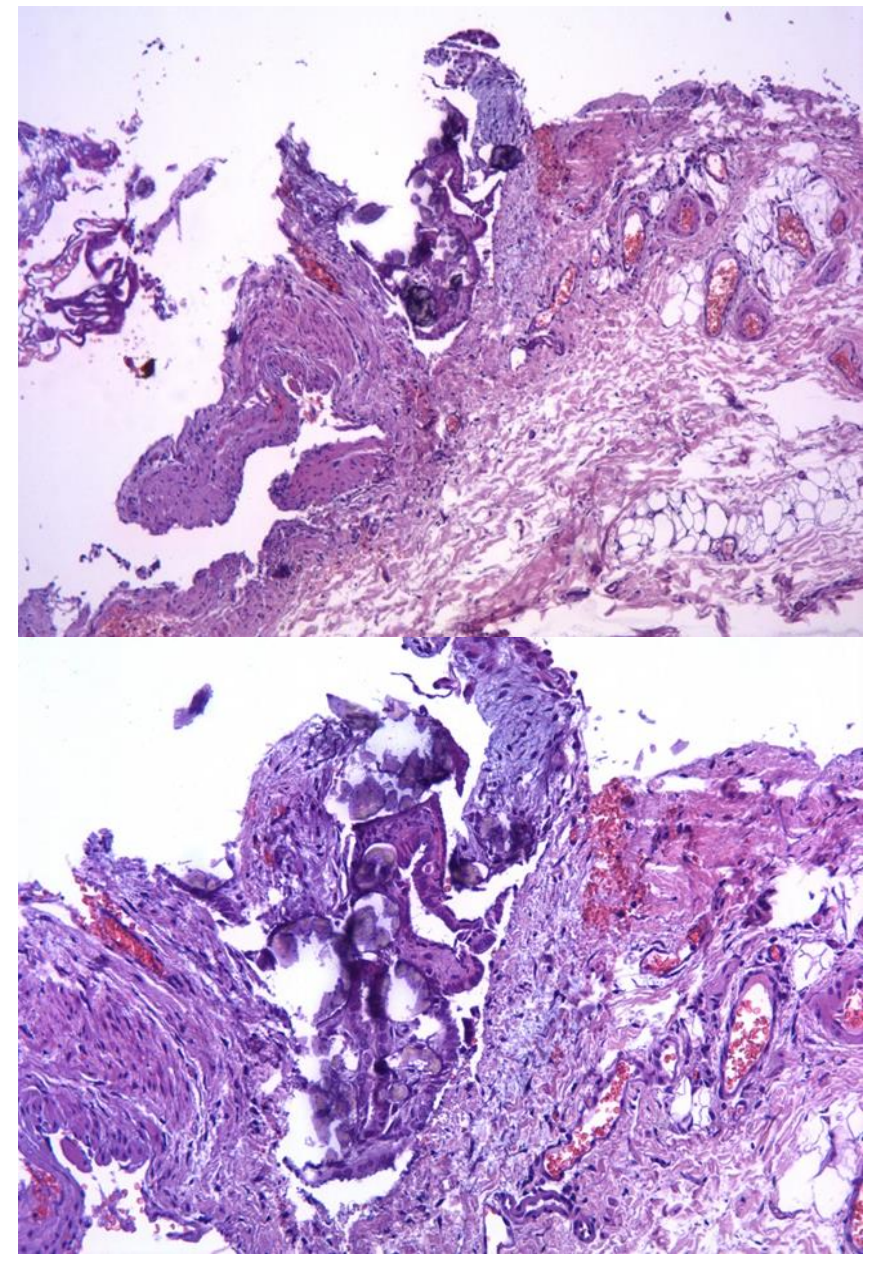

Figure 2. PPSBT: Hematoxylline-eosine staining noninvasive epithelial implant of serous borderline tumor. Papillae with slightly atypical epithelial cell and proeminent psammoma bodies (HE 5x, HE 10x)

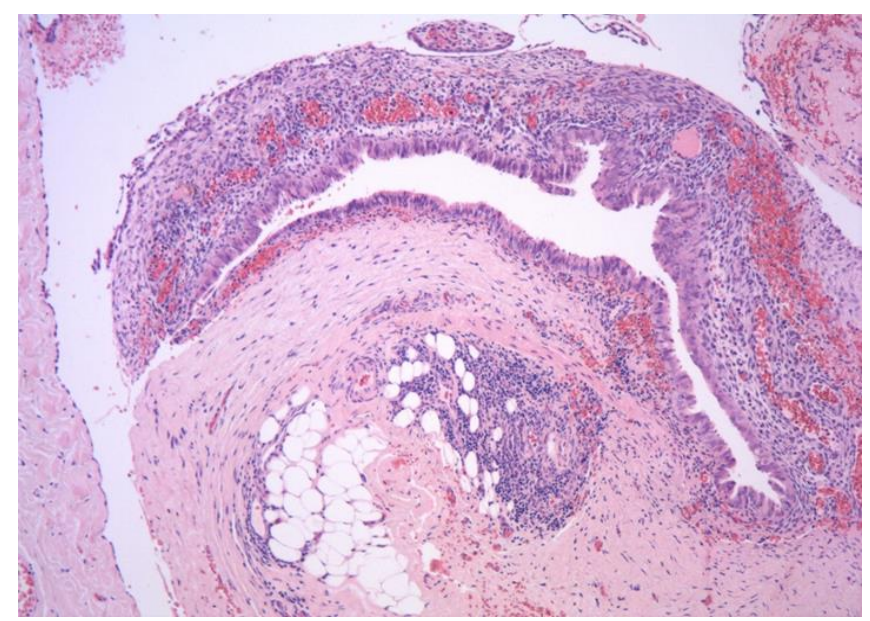

Figure 3. Endometriosis : peritoneal inclusion of ectopic endometriotic like gland and stroma (HE 10x)

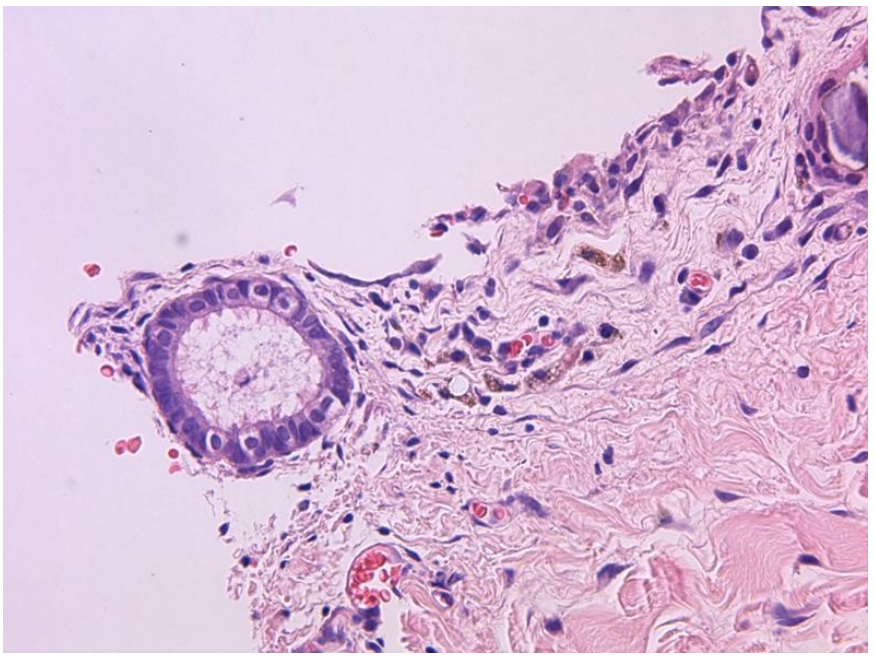

Figure 4. Endosalpingiosis: peritoneal inclusion of tubal type gland lined by a single layer of ciliated cell (HE 40x)

\section{Discussion and Conclusion}

We report here the case of a rare association of PPSBT and endometriosis found during surgery for CPP. Although PPSBT is a rare entity, macroscopic aspects cannot distinguish PPSBT from other benign or malignant lesion. The prognosis and the treatment of each histological entity are completely different.

Our case highlights the absolute importance of pathological analyses of the peritoneal lesion and the careful examination of both ovaries, regardless of the preoperative indication or the surgical technique used. During eradication of endometriosis, even if the lesions seem to have a macroscopic benign typical aspect, biopsies are always mandatory, especially if the surgeon use an ablation technique.

\section{References}

1. Brichant G, Denef M, Tebache L, Poismans G, Pinzauti S, et al. Chronic pelvic pain and the role of exploratory laparoscopy as diagnostic and therapeutic tool: a retrospective observational study. 2018; 13.

2. Garry R. The effectiveness of laparoscopic excision of endometriosis. Curr Opin Obstet Gynecol. 2004; 16: 299-303.

3. Lamvu G, Tu F, As-Sanie S, Zolnoun D, Steege JF. The role of laparoscopy in the diagnosis and treatment of conditions associated with chronic pelvic pain. Obstet Gynecol Clin North Am. 2004; 31: 619-630.

4. deHoop TA, Mira J, Thomas MA. Endosalpingiosis and chronic pelvic pain. J Reprod Med. 1997; 42: 613-616.

5. Heinig J, Gottschalk I, Cirkel U, Diallo R. Endosalpingiosis-an underestimated cause of chronic pelvic pain or an accidental finding? A retrospective study of 16 cases. Eur J Obstet Gynecol Reprod Biol. 2002; 103: 75-78.

6. Fox H. Primary neoplasia of the female peritoneum. Histopathology. 1993; 23: 103-110.

7. Hutton RL, Dalton SR. Primary peritoneal serous borderline tumors. Arch Pathol Lab Med. 2007; 131: 138-144.

8. Nicosia SV. Ovarian and Peritoneal Borderline Neoplasms: Histopathology, Diagnostic Pitfalls, and Prognostication. Cancer Control. 1996; 3: 58-65. 
9. Biscotti CV, Hart WR. Peritoneal serous micropapillomatosis of low malignant potential (serous borderline tumors of the peritoneum). A clinicopathologic study of 17 cases. Am J Surg Pathol. 1992; 16: 467-475.
10. Bell DA, Scully RE. Serous borderline tumors of the peritoneum. Am J Surg Pathol. 1990; 14: 230-239.

*Correspondence: Linda Tebache, Department of Obstetrics and Gynaecology, University of Liège, CHR Liège, Boulevard du 12ème De Ligne, 4000 Liège, Belgium, Tel: 00324321 6582, E-mail: linda.tebache@chrcitadelle.be

Rec: Mar 25, 2020; Acc: Apr 16, 2020; Pub: Apr 18, 2020

Arch Obs Gyn. 2020;1(2):109

DOI: gsl.aog.2020.000109

Copyright (C) 2020 The Author(s). This is an open-access article distributed under the terms of the Creative Commons Attribution 4.0 International License (CC-BY). 